\title{
Computing special values of partial zeta functions
}

\author{
Gautam Chinta $^{1}$, Paul E. Gunnells ${ }^{1}$, and Robert Sczech ${ }^{2}$ \\ 1 Dept. of Mathematics, Columbia University, New York, NY 10027, USA \\ 2 Dept. of Mathematics and Computer Science, Rutgers University, Newark, NJ \\ 07102-1811, USA
}

\begin{abstract}
We discuss computation of the special values of partial zeta functions associated to totally real number fields. The main tool is the Eisenstein cocycle $\Psi$, a group cocycle for $G L_{n}(\mathbb{Z})$; the special values are computed as periods of $\Psi$, and are expressed in terms of generalized Dedekind sums. We conclude with some numerical examples for cubic and quartic fields of small discriminant.
\end{abstract}

\section{Introduction}

Let $K / \mathbb{Q}$ be a totally real number field of degree $n$ with ring of integers $\mathscr{O}_{K}$, and let $U \subset \mathscr{O}_{K}^{\times}$be the subgroup of totally positive units. Let $\mathfrak{f}, \mathfrak{b} \subset \mathscr{O}_{K}$ be relatively prime ideals. Then the partial zeta function associated to this data is defined by

$$
\zeta_{\mathfrak{f}}(\mathfrak{b}, s):=\sum_{\mathfrak{a} \sim \mathfrak{b}} N(\mathfrak{a})^{-s},
$$

where $\mathfrak{a} \sim \mathfrak{b}$ means $\mathfrak{a b}^{-1}=(\alpha)$, where $\alpha$ is a totally positive number in $1+\mathfrak{f b}^{-1}$. According to a classical result of Klingen and Siegel, the special values $\zeta_{\mathfrak{f}}(\mathfrak{b}, k)$ are rational for nonpositive integers $k$. Moreover, the values $\zeta_{\mathfrak{f}}(\mathfrak{b}, 0)$ are especially important because of their connection with the Brumer-Stark conjecture and the Leopoldt conjecture $[7,6,3,8,10]$.

In [9], one of us (RS) gave a cohomological interpretation of these special values by showing that they can be computed in finite terms as periods of the Eisenstein cocycle. This is a cocycle $\Psi \in H^{n-1}\left(G L_{n}(\mathbb{Z}) ; \mathscr{M}\right)$, where $\mathscr{M}$ is a certain $G L_{n}(\mathbb{Z})$-module. Then two of us (PEG and RS) showed in [5] that the Eisenstein cocycle is an effectively computable object. More precisely, using the cocycle one can express $\zeta_{\mathfrak{f}}(\mathfrak{b}, k)$ as a finite sum of generalized Dedekind sums, and that the latter can be effectively computed by a continued-fraction algorithm that uses a generalization of the classical Dedekind-Rademacher reciprocity law.

In this note we describe an ongoing project to build a database of $\zeta_{\mathfrak{f}}(\mathfrak{b}, 0)$ for various fields $K$ and ideals $\mathfrak{f}, \mathfrak{b}$. We recall the definition of the Eisenstein cocycle and its relation to the special values $(\S 2)$, and discuss the effective computation of Dedekind sums (§3). We conclude with examples of special values for some fields of degree 3 and $4(\S 4)$. 


\section{Dedekind sums and the Eisenstein cocycle}

\section{1}

Let $\sigma$ be a square matrix with integral columns $\sigma_{j} \in \mathbb{Z}^{n}(j=1, \ldots, n)$, and let $L \subset \mathbb{Z}^{n}$ be a lattice of rank $r \geq 1$. Let $v \in \mathbb{Q}^{n}$, and let $e \in \mathbb{Z}^{n}$ with $e_{j} \geq 1$. Then the Dedekind sum $S$ associated to the data $(L, \sigma, e, v)$ is defined by

$$
S=S(L, \sigma, e, v):=\sum_{x \in L}^{\prime} \mathbf{e}(\langle x, v\rangle) \frac{\operatorname{det} \sigma}{\left\langle x, \sigma_{1}\right\rangle^{e_{1}} \cdots\left\langle x, \sigma_{n}\right\rangle^{e_{n}}} .
$$

Here $\langle x, y\rangle:=\sum x_{i} y_{i}$ is the usual scalar product on $\mathbb{R}^{n}, \mathbf{e}(t)$ is the character $\exp (2 \pi i t)$, and the prime next to the summation means to omit terms for which the denominator vanishes. The series (1) converges absolutely if all $e_{j}>1$, but may only converge conditionally if $e_{j}=1$ for some $j$. In this latter case we can define the sum by the $Q$-limit

$$
\left.\sum_{x \in L}^{\prime} a(x)\right|_{Q}:=\lim _{t \rightarrow \infty}\left(\sum_{|Q(x)|<t}^{\prime} a(x)\right)
$$

where $Q$ is any finite product of real-valued linear forms on $\mathbb{R}^{n}$ that doesn't vanish on $\mathbb{Q}^{n} \backslash\{0\}$. One can precisely determine how the value of $(1)$ depends on $Q([9$, Thm. 7$])$. The sum $S$ is always a rational number times a power of $2 \pi i$.

\section{2}

We recall now the definition of the Eisenstein cocycle $\Psi$ and its relationship with the special values $\zeta_{\mathfrak{f}}(\mathfrak{b}, k)$. For simplicity, we describe only material necessary to compute the special value at $k=0$, and refer to $[9,5]$ for other $k$.

Let $\mathscr{A}=\left(A_{1}, \ldots, A_{n}\right) \in\left(G L_{n}(\mathbb{R})\right)^{n}$ be an $n$-tuple of matrices. For an $n$ tuple $d=\left(d_{1}, \ldots, d_{n}\right)$ of integers $1 \leq d_{i} \leq n$, let $\mathscr{A}(d) \subseteq \mathbb{R}^{n}$ be the subspace generated by all columns $A_{i j}$ such that $j<d_{i}$. (Here $A_{i j}$ denotes the $j$ th column of the matrix $A_{i}$.) Writing $\mathscr{A}(d)^{\perp}$ for the orthogonal complement of $\mathscr{A}(d)$ in $\mathbb{R}^{n}$, we let

$$
X(d)=\mathscr{A}(d)^{\perp} \backslash \bigcup_{i=1}^{n} \sigma_{i}^{\perp}, \quad \text { where } \sigma_{i}=A_{i d_{i}} .
$$

The $n$-tuple $\mathscr{A}$ determines a decomposition of $\mathbb{R}^{n} \backslash\{0\}$ into linear strata

$$
\bigsqcup_{d \in D} X(d)
$$

indexed by the finite set

$$
D=D(\mathscr{A})=\{d \mid X(d) \neq \varnothing\} .
$$


Associated to this decomposition is a collection of rational functions $\psi(\mathscr{A})$ on $\mathbb{R}^{n} \backslash\{0\}$, defined by

$$
\psi(\mathscr{A})(x)=\frac{\operatorname{det}\left(\sigma_{1}, \ldots, \sigma_{n}\right)}{\left\langle x, \sigma_{1}\right\rangle \cdots\left\langle x, \sigma_{n}\right\rangle}, \quad \text { if } x \in X(d) .
$$

Note that $\psi(\mathscr{A})(x)$ is well-defined by the construction of $X(d)$.

Let $v \in \mathbb{R}^{n}$, and let $Q$ be defined as in $\S 2.1$. Then the Eisenstein cocycle $\Psi$ is defined as

$$
\Psi=\Psi(\mathscr{A})(Q, v):=\left.(2 \pi i)^{-n} \sum_{x \in \mathbb{Z}^{n}} \mathbf{e}(\langle x, v\rangle) \psi(\mathscr{A})(x)\right|_{Q} .
$$

One can show that $\Psi$ is a homogeneous $(n-1)$-cocycle for $G L_{n}(\mathbb{Z})$. Furthermore, we can express $\Psi$ in terms of Dedekind sums

$$
\Psi(\mathscr{A})(Q, v)=\left.(2 \pi i)^{-n} \sum_{d \in D} S(L(d), \sigma, \mathbf{1}, v)\right|_{Q},
$$

where $\sigma$ is the matrix with columns $A_{i d_{i}},(i=1, \ldots, n), L(d)$ is the lattice $\mathscr{A}(d)^{\perp} \cap \mathbb{Z}^{n}$, and $\mathbf{1}$ is the vector $(1, \ldots, 1)$.

\section{3}

Now we describe how $\Psi$ can be used to compute special values. Let $W$ be a $\mathbb{Z}$-basis for the fractional ideal $\mathfrak{f b}^{-1}=\sum \mathbb{Z} W_{j}$, and let $W^{*}$ be the dual basis with respect to the trace form. Via the $n$ real embeddings $\tau_{i}, i=1, \ldots, n$, any $x \in K$ determines a row vector $\left(\tau_{1}(x), \ldots, \tau_{n}(x)\right)$. Hence we may identify $W$ with a matrix in $G L_{n}(\mathbb{R})$ : the $j$ th row of this matrix is the image of the $j$ th basis element of $W$. Let

$$
Q(X)=\prod_{i} \sum_{j} X_{j}\left(\tau_{i}\left(W_{j}^{*}\right)\right)
$$

and let $v \in \mathbb{Q}^{n}$ be defined by $v_{j}=\operatorname{Tr}\left(W_{j}^{*}\right)$.

Let $\nu=n-1$, and let $\varepsilon_{1}, \ldots, \varepsilon_{\nu}$ be a basis for the totally positive units $U$. Using the regular representation $\rho$ with respect to the basis $W$, we identify the units $\varepsilon_{j}$ with elements $A_{j}=\rho\left(\varepsilon_{j}\right)^{t} \in G L_{n}(\mathbb{Z})$. Using the bar notation

$$
\left[A_{1}|\cdots| A_{\nu}\right]:=\left(1, A_{1}, A_{1} A_{2}, \ldots, A_{1} \cdots A_{\nu}\right) \in\left(G L_{n}(\mathbb{Z})\right)^{n},
$$

we have the following proposition expressing the zeta values in terms of the Eisenstein cocycle:

Proposition 1. [9, 5] Let $U_{\mathfrak{f}}$ be the subgroup $U \cap(1+\mathfrak{f})$, and let $\pi$ run through all permutations of $\{1, \ldots, \nu\}$. Then

$$
\zeta_{\mathfrak{f}}(\mathfrak{b}, 0)=\eta \sum_{\varepsilon \in U / U_{\mathfrak{f}}} \sum_{\pi} \operatorname{sgn}(\pi) \Psi\left(\left[A_{\pi(1)}|\cdots| A_{\pi(\nu)}\right]\right)\left(Q, \rho(\varepsilon)^{t} v\right) .
$$

Here $\eta= \pm 1$ is defined by

$$
\eta=(-1)^{\nu} \operatorname{sgn}(\operatorname{det} W) \operatorname{sgn}(R),
$$

where $R=\operatorname{det}\left(\log \tau_{j}\left(\varepsilon_{i}\right)\right), 1 \leq i, j \leq \nu$. 


\section{Diagonality and unimodularity}

\section{1}

We define the rank of $S=S(L, \sigma, e, v)$ to be the rank of the lattice $L$. It is easy to see that after a $G L_{n}(\mathbb{Q})$ transformation, we may assume that $L$ is the sublattice $Z^{\ell}$ spanned by the first $\ell$ standard basis vectors, where $\ell$ is the rank of $L$. Furthermore, by multiplying by an appropriate rational factor, permuting columns and repeating columns if necessary, we may assume the pair $\left(Z^{\ell}, \sigma\right)$ satisfies the following conditions:

(i) For each column $\sigma_{j}$, the vector of the first $\ell$ components of $\sigma_{j}$ is primitive and integral.

(ii) If two columns of $\sigma$ induce proportional linear forms on $Z^{\ell}$, then these two linear forms coincide on $Z^{\ell}$, and are adjacent columns of $\sigma$.

(iii) The vector $e=\mathbf{1}$.

Let $S\left(Z^{\ell}, \sigma, \mathbf{1}, v\right)$ be a Dedekind sum satisfying the three conditions above. Let $\pi: \mathbb{R}^{N} \rightarrow \mathbb{R}^{\ell}$ be the projection on the first $\ell$ components, and let $\pi(\sigma)$ be the $\ell \times n$ matrix with columns $\pi\left(\sigma_{i}\right)$.

Definition 1. Let $M(\sigma)$ be the set of maximal minors of $\pi(\sigma)$. Then the index of $S$, denoted $\|S\|$, is defined to be

$$
\max _{\tau \in M(\sigma)}|\operatorname{det} \tau|
$$

A Dedekind sum is unimodular if $\|S\|=1$.

\section{2}

Now define a partition

$$
\llbracket n \rrbracket=\bigsqcup_{k=1}^{s} I_{k}, \quad \ell \leq s \leq n
$$

as follows. Put

$$
i, j \in I_{k} \quad \text { if and only if } \quad \pi\left(\sigma_{i}\right)=\pi\left(\sigma_{j}\right) \text {. }
$$

In other words, two elements of $\llbracket n \rrbracket$ are in the same set of the partition if the corresponding columns of $\sigma$ induce the same linear form on $Z^{\ell}$.

Let $p_{k}=\# I_{k}$.

Definition 2. The vector $p(S)=\left(p_{1}, \ldots, p_{s}\right)$ is called the type of $S$. A Dedekind sum is called diagonal if $p(S)$ has length $\ell$. 


\section{3}

The virtue of diagonality is that a diagonal Dedekind sum $S$ may be evaluated as a finite sum of products of generalized Bernoulli polynomials. Furthermore, the number of terms in this finite sum is the index of $S$. Hence diagonal and unimodular Dedekind sums can be evaluated very rapidly.

In general, the Dedekind sums in (5) aren't diagonal. However, we have the following theorem, which is the main result of [5]:

Theorem 1. [5] Every Dedekind sum $S(L, \sigma, e, v)$ can be expressed as a finite rational linear combination of unimodular diagonal sums. If $n$, Rank $L$, and $e$ are fixed, then this expression can be computed in time polynomial in log $\|S\|$. Moreover, the number of terms in this expression is bounded by a polynomial in $\log \|S\|$.

The key ingredient in the proof of Theorem 1 is a "reciprocity law" for higher-dimensional Dedekind sums. For any nonzero point $v \in \mathbb{R}^{n}$, let $v^{\perp}$ be the hyperplane $\{x \mid\langle v, x\rangle=0\}$. Let $Q$ be a finite product of real-valued linear forms on $\mathbb{R}^{n}$ that do not vanish on $\mathbb{Q}^{n} \backslash\{0\}$.

Proposition 2. Let $\sigma_{0}, \ldots, \sigma_{n} \in \mathbb{Z}^{n}$ be nonzero. For $j=0, \ldots, n$, let $\sigma^{j}$ be the matrix with columns $\sigma_{0}, \ldots, \hat{\sigma}_{j}, \ldots, \sigma_{n}$. Fix a lattice $L \subseteq \mathbb{Z}^{n}$, and assume $e=\mathbf{1}$. Then for any $v \in \mathbb{R}^{n}$, we have the following identity among Dedekind sums:

$$
\left.\sum_{j=0}^{n}(-1)^{j} S\left(L, \sigma^{j}, \mathbf{1}, v\right)\right|_{Q}=\left.\sum_{j=0}^{n}(-1)^{j} S\left(L \cap \sigma_{j}^{\perp}, \sigma^{j}, \mathbf{1}, v\right)\right|_{Q} .
$$

We refer to [5] for proofs of the above statements. Here, in the following two sections, we show how Theorem 1 is applied with a rank 2 example. For simplicity we ignore issues of convergence, and merely remark that all of our manipulations with sums are compatible with the $Q$-limit process (2).

\section{4}

Let $L$ be the lattice $Z^{2}$. Let

$$
\sigma=\left(\begin{array}{lll}
1 & 0 & 1 \\
0 & 1 & 2 \\
0 & 0 & 1
\end{array}\right), \quad e=(1,1,2), \quad \text { and } v=(0,0,0)
$$

Hence $S(L, \sigma, e, v)$ denotes the absolutely convergent sum

$$
\sum_{(x, y) \in \mathbb{Z}^{2}}^{\prime} \frac{1}{x y(x+2 y)^{2}}
$$

where the prime on the summation indicates that we omit the terms $(x, y)$ for which $x, y$ or $x+2 y$ vanish. 
This sum isn't diagonal, since $\sigma$ induces 3 different linear forms on $L$ instead of 2 . To diagonalize $S$, we begin with the identity of rational functions

$$
\frac{1}{x y(x+2 y)^{2}}=\frac{1}{y(x+2 y)^{3}}+\frac{2}{x(x+2 y)^{3}} .
$$

This is true provided none of the denominators vanishes. The numerators of the functions on the right come from expressing the third column of $\sigma$ as a linear combination of the first two:

$$
(1,2)^{t}=1 \cdot(1,0)^{t}+2 \cdot(0,1)^{t} .
$$

We want to sum both sides of (8) over pairs $(x, y) \in \mathbb{Z}^{2}$ to obtain an identity among Dedekind sums of the form

$$
\sum_{(x, y) \in \mathbb{Z}^{2}}^{\prime} \frac{1}{x y(x+2 y)^{2}}=\sum_{(x, y) \in \mathbb{Z}^{2}}^{\prime} \frac{1}{y(x+2 y)^{3}}+\sum_{(x, y) \in \mathbb{Z}^{2}}^{\prime} \frac{2}{x(x+2 y)^{3}} .
$$

However, as written (9) is incorrect. The identity (8) only holds if none of $x, y$, or $x+2 y$ vanish, but the sums on the right of (9) include some of these terms (for instance, the first sum on the right of (9) contains terms $(x, y)$ with $x=0$ ). We account for this by subtracting two rank 1 Dedekind sums from the right of (9) as "correction terms":

$$
\begin{aligned}
& \sum_{(x, y) \in \mathbb{Z}^{2}}^{\prime} \frac{1}{x y(x+2 y)^{2}}=\sum_{(x, y) \in \mathbb{Z}^{2}}^{\prime} \frac{1}{y(x+2 y)^{3}}+\sum_{(x, y) \in \mathbb{Z}^{2}}^{\prime} \frac{2}{x(x+2 y)^{3}} \\
& -\sum_{\substack{(x, y) \in \mathbb{Z}^{2} \\
x=0}}^{\prime} \frac{1}{y(x+2 y)^{3}}-\sum_{\substack{(x, y) \in \mathbb{Z}^{2} \\
y=0}}^{\prime} \frac{1}{x(x+2 y)^{3}} \\
& =\quad \sum_{(x, y) \in \mathbb{Z}^{2}}^{\prime} \frac{1}{y(x+2 y)^{3}}+\sum_{(x, y) \in \mathbb{Z}^{2}}^{\prime} \frac{2}{x(x+2 y)^{3}} \\
& -\sum_{y \in \mathbb{Z}}^{\prime} \frac{1}{8 y^{4}} \quad-\sum_{x \in \mathbb{Z}}^{\prime} \frac{2}{x^{4}} \text {. }
\end{aligned}
$$

This equation is precisely an instance of the reciprocity law (Proposition 2). The three rank 2 sums are the left of (7), and the two rank 1 sums are the right of (7) (one rank 1 sum in (7) vanishes identically). Note that all of the sums on the right of (10) are now diagonal.

To diagonalize a general Dedekind sum $S(L, \sigma, \mathbf{1}, v)$, one considers the configuration $C \subset \mathbb{R}^{n}$ of linear subspaces consisting of $(L \otimes \mathbb{R})^{\perp}$ and the spaces generated by the points $\sigma_{1}, \ldots, \sigma_{n}$. One shows by investigating the geometry of $C$ that a point $\sigma_{0}$ can be found such that when Proposition 2 is applied with the tuple $\left(\sigma_{0}, \ldots, \sigma_{n}\right)$, the resulting Dedekind sums are "closer" to diagonality in a certain sense. It may take several applications of Proposition 2 to express a Dedekind sum as a linear combination of diagonal sums. 


\section{5}

The second rank two sum on the right of (10) has index two. We will show how to make this sum unimodular. Write

$$
S\left(\mathbb{Z}^{2}, \tau,(1,3), v^{\prime}\right)=\sum_{(x, y) \in \mathbb{Z}^{2}}^{\prime} \frac{1}{x(x+2 y)^{3}}
$$

where

$$
\tau=\left(\tau_{1}, \tau_{2}\right)=\left(\begin{array}{ll}
1 & 1 \\
0 & 2
\end{array}\right) \quad \text { and } \quad v^{\prime}=(0,0) .
$$

Let $\rho$ be the column vector $(0,1)^{t}$. We apply Proposition 2 to the triple $\left(\rho, \tau_{1}, \tau_{2}\right)$ :

$$
\begin{aligned}
\sum_{(x, y) \in \mathbb{Z}^{2}}^{\prime} \frac{2}{x(x+2 y)^{3}}= & \sum_{(x, y) \in \mathbb{Z}^{2}}^{\prime} \frac{-1}{y(x+2 y)^{3}}+\sum_{(x, y) \in \mathbb{Z}^{2}}^{\prime} \frac{1}{x(x+2 y)^{2} y} \\
& +\sum_{y \in \mathbb{Z}}^{\prime} \frac{1}{8 y^{4}}+\sum_{x \in \mathbb{Z}}^{\prime} \frac{2}{x^{4}} .
\end{aligned}
$$

Now all the terms on the right of (11) are diagonal and unimodular except for the second rank two sum. In fact, this sum is no longer diagonal. However, one further application of Proposition 2 as in $\S 3.4$ will make the third sum diagonal and unimodular. Hence we will have succeeded in expressing the original sum as a finite linear combination of diagonal, unimodular Dedekind sums.

In general, one must be able to construct the vector $\rho$ as above. An easy argument using Minkowski's Theorem from the geometry of numbers guarantees the existence of $\rho[1]$. To construct $\rho$ in practice, one may use $L L L$-reduction of the lattice spanned by the rows of $\sigma$ and [4, Conjecture 3.9].

\section{Examples}

Here we present some numerical examples. For simplicity we compute $\zeta=$ $\zeta_{\mathfrak{f}}(\mathfrak{b}, 0)$, where $\mathfrak{f}=N \mathscr{O}_{K}$ for various rational integers $N$, and $\mathfrak{b}=\mathscr{O}_{K}$. These fields are the first entries in the tables of totally real fields with small discriminant, available from [2].

Cubic fields

- $K=\mathbb{Q}(\theta)$, where $\theta^{3}+\theta^{2}-2 \theta-1=0$ (discriminant 49$)$.

\begin{tabular}{|r|r||r|r||r|r||r|r||r|r||r|r||r|r||r|r|}
\hline$N$ & $N \cdot \zeta$ & $N$ & $N \cdot \zeta$ & $N$ & $N \cdot \zeta$ & $N$ & $N \cdot \zeta$ & $N$ & $N \cdot \zeta$ & $N$ & $N \cdot \zeta$ & $N$ & $N \cdot \zeta$ & $N$ & $N \cdot \zeta$ \\
\hline \hline & & 4 & 1 & 7 & 2 & 10 & 16 & 13 & -1 & 16 & -9 & 19 & 18 & 22 & -8 \\
2 & 0 & 5 & 2 & 8 & 3 & 11 & -10 & 14 & -24 & 17 & -26 & 20 & 19 & 23 & 10 \\
3 & 2 & 6 & 8 & 9 & 2 & 12 & 5 & 15 & 14 & 18 & 8 & 21 & 6 & 24 & 23 \\
\hline
\end{tabular}


- $K=\mathbb{Q}(\theta)$, where $\theta^{3}-3 \theta-1=0$ (discriminant 81$)$.

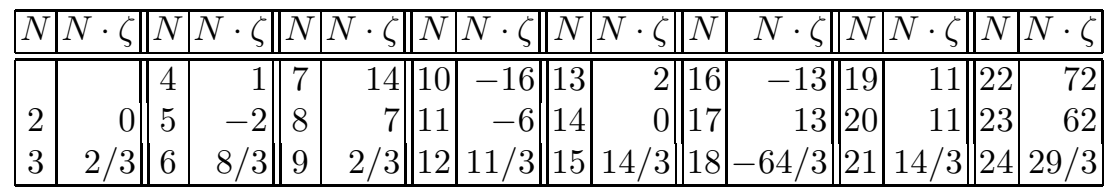

- $K=\mathbb{Q}(\theta)$, where $\theta^{3}+\theta^{2}-3 \theta-1=0$ (discriminant 148).

\begin{tabular}{|c|c|c|c|c|c|c|c|c|c|c|c|c|c|c|}
\hline 1 & & $N$ & $\underline{\zeta}$ & $\| N$ & $\zeta$ & $N$ & $N$ & $N$ & $\cdot \zeta$ & $N$ & $\cdot \zeta \| I$ & & & \\
\hline & & 4 & $\overline{1}$ & $\mid \overline{7}$ & & 10 & $\overline{-2}$ & $\mid 13$ & -22 & 16 & \begin{tabular}{l||l|}
7 & 19
\end{tabular} & & 22 & 68 \\
\hline 2 & 0 & 5 & -4 & 8 & & 11 & -18 & $\mid 14$ & -20 & 17 & 100|| 20 & & 4|| 23 & 12 \\
\hline 2 & & 6 & & $\mid$ | 9 & -10 & $12 \mid$ & & 15 & 42 & 18 & -32|| 21 & -78 & || 24 & 2 \\
\hline
\end{tabular}

- $K=\mathbb{Q}(\theta)$, where $\theta^{3}-\theta^{2}-4 \theta-1=0$ (discriminant 169 ).

\begin{tabular}{|r|r||r|r||r|r||r|r||r|r||r|r||r|r||r|r|}
\hline$N$ & $N \cdot \zeta$ & $N$ & $N \cdot \zeta$ & $N$ & $N \cdot \zeta$ & $N$ & $N \cdot \zeta$ & $N$ & $N \cdot \zeta$ & $N$ & $N \cdot \zeta$ & $N$ & $N \cdot \zeta$ & $N$ & $N \cdot \zeta$ \\
\hline \hline & & 4 & 3 & 7 & 6 & 10 & 8 & 13 & 2 & 16 & 25 & 19 & -238 & 22 & 160 \\
2 & 0 & 5 & 1 & 8 & 5 & 11 & -6 & 14 & 56 & 17 & 6 & 20 & -8 & 23 & 386 \\
3 & 2 & 6 & 8 & 9 & 26 & 12 & 11 & 15 & 17 & 18 & 52 & 21 & -10 & 24 & 89 \\
\hline
\end{tabular}

- $K=\mathbb{Q}(\theta)$, where $\theta^{3}-4 \theta-1=0$ (discriminant 229).

\begin{tabular}{|l|r||r|r||r|r||r|r||r|r||r|r||r|r||r|r|}
\hline$N$ & $N \cdot \zeta$ & $N$ & $N \cdot \zeta$ & $N$ & $N \cdot \zeta$ & $N$ & $N \cdot \zeta$ & $N$ & $N \cdot \zeta$ & $N$ & $N \cdot \zeta$ & $N$ & $N \cdot \zeta$ & $N$ & $N \cdot \zeta$ \\
\hline \hline & & 4 & 2 & 7 & 18 & 10 & -24 & 13 & 60 & 16 & 19 & 19 & 26 & 22 & 8 \\
2 & 0 & 5 & -2 & 8 & 7 & 11 & -4 & 14 & 24 & 17 & 8 & 20 & 38 & 23 & 202 \\
3 & 2 & 6 & 0 & 9 & -10 & 12 & 18 & 15 & 56 & 18 & -16 & 21 & 96 & 24 & 51 \\
\hline
\end{tabular}

- $K=\mathbb{Q}(\theta)$, where $\theta^{3}-\theta^{2}-4 \theta+3=0$ (discriminant 257).

\begin{tabular}{|c|c|c|c|c|c|c|c|c|c|c|c|c|c|c|c|}
\hline V & $N \cdot \zeta$ & $\| N \mid 1$ & & $\| N$ & & ||$N \mid$ & $N \cdot \zeta$ & $\zeta \| N$ & $N \cdot \zeta$ & $N$ & $N \cdot \zeta$ & $\| N$ & $N \cdot \zeta$ & $\| N$ & $N$ \\
\hline & & $\mid 4$ & & $\mid 17$ & & \begin{tabular}{|l||l|}
5 & 10
\end{tabular} & & \begin{tabular}{l|l|l}
4 & 13
\end{tabular} & 10 & 16 & & || $\mid 19$ & 42 & $\| 22$ & $\overline{7}$ \\
\hline 2 & & || 5 & & 8 & & 9 & -6 & \begin{tabular}{l||l}
6 & 1
\end{tabular} & 0 & 17 & -26 & $\| 20$ & 12 & $\| 23$ & -11 \\
\hline & 2 & $\| 0$ & & 5 & 4 & \begin{tabular}{l||l|}
4 & 12
\end{tabular} & & 8|| $1:$ & 21 & 18 & -16 & $\mid 21$ & -12 & $\mid 24$ & 5 \\
\hline
\end{tabular}

Quartic fields

- $K=\mathbb{Q}(\theta)$, where $\theta^{4}-\theta^{3}-3 \theta^{2}+\theta+1=0$ (discriminant 725).

\begin{tabular}{|c|c|c|c|c|c|c|c|c|c|c|c|c|c|c|}
\hline & & $N$ & & $N$ & \begin{tabular}{l||l|}
$\cdot \zeta$ \\
\end{tabular} & $\zeta$ & $|N|$ & $N \cdot \zeta$ & & $N \cdot \zeta$ & & 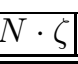 & & $\cdot$ \\
\hline & & 4 & 1 & 7 & \begin{tabular}{l||l|}
-4 & 10
\end{tabular} & $\overline{\overline{0}}$ & $\mid 13$ & \begin{tabular}{c|}
-20 \\
\end{tabular} & 16 & 35 & || 19 & \begin{tabular}{|c|}
-32 \\
\end{tabular} & 22 & \\
\hline 2 & & 5 & & 8 & $7 \| 11$ & -2 & || 14 & -16 & 17 & -92 & $\mid 20$ & 0 & $\mid 23$ & - \\
\hline 3 & & 10 & & 9 & -44|| 12 & & 15 & & & -320 & ||21 & -30 & || 24 & -8 \\
\hline
\end{tabular}


- $K=\mathbb{Q}(\theta)$, where $\theta^{4}-\theta^{3}-4 \theta^{2}+4 \theta+1=0$ (discriminant 1125).

\begin{tabular}{|c|c|c|c|c|c|c|c|c|c|c|c|c|}
\hline & $N \cdot \zeta|| \Lambda$ & \begin{tabular}{l|l||}
$N$ & $N \cdot \zeta$
\end{tabular} & $N|N \cdot \zeta| \mid$ & & $N \cdot \zeta$ & $\| N$ & $N \cdot \zeta$ & \begin{tabular}{||l|l}
$|N|$ & $\Lambda$ \\
\end{tabular} & $\overline{N \cdot \zeta \| N}$ & $\sqrt{T}$ & $N$ & $\cdot 6$ \\
\hline & & \begin{tabular}{l||l}
$4 / 5$ & 5
\end{tabular} & $\overline{4 / 3}$ & & $124 / 3$ & 9 & $\overline{1 / 5}$ & & 72 & 6 & & \\
\hline & 0 & 2|| 6 & 6 & & & $\|$ & 128 & 12 & & 4 & & \\
\hline
\end{tabular}

- $K=\mathbb{Q}(\theta)$, where $\theta^{4}-6 \theta^{2}+4=0$ (discriminant 1600).

\begin{tabular}{|c|c|c|c|c|c|c|c|c|c|c|c|c|c|c|c|}
\hline & & $N$ & $\zeta$ & $\| N$ & $N \cdot \zeta$ & $\| N$ & $\cdot \zeta$ & $\| N$ & $N \cdot \zeta$ & $|N|$ & $N \cdot \zeta$ & $\| \Lambda$ & $N \cdot \zeta$ & $\| N$ & $\bar{N}$. \\
\hline & & 4 & 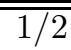 & $\mid 77$ & & $\| 10$ & 4 & 13 & $\overline{104}$ & 16 & $\mid 45 / 2$ & 19 & $\overline{\overline{224}}$ & 22 & $\overline{\overline{136}}$ \\
\hline 2 & 1 & 5 & 4 & 8 & $5 / 2$ & $\| 11$ & -4 & 14 & -16 & || 17 & -84 & 20 & -9 & $\mid 23$ & 60 \\
\hline 3 & & 6 & & || 9 & 16 & $\mid 12$ & 24 & 15 & 6 & $\mid 18$ & 128 & 21 & 222 & 24 & 1. \\
\hline
\end{tabular}

- $K=\mathbb{Q}(\theta)$, where $\theta^{4}-4 \theta^{2}-\theta+1=0$ (discriminant 1957).

\begin{tabular}{|r|r||r|r||r|r||r|r||r|r||r|r||r|r|}
\hline$N$ & $N \cdot \zeta$ & $N$ & $N \cdot \zeta$ & $N$ & $N \cdot \zeta$ & $N$ & $N \cdot \zeta$ & $N$ & $N \cdot \zeta$ & $N$ & $N \cdot \zeta$ & $N$ & $N \cdot \zeta$ \\
\hline \hline & & 3 & 4 & 5 & 20 & 7 & -8 & 9 & 52 & 11 & 4 & 13 & 500 \\
2 & 0 & 4 & 1 & 6 & 0 & 8 & 11 & 10 & 0 & 12 & 3 & 14 & -8 \\
\hline
\end{tabular}

- $K=\mathbb{Q}(\theta)$, where $\theta^{4}-5 \theta^{2}+5=0$ (discriminant 2000).

\begin{tabular}{|c|c|c|c|c|c|c|c|c|c|c|c|c|c|c|}
\hline \multicolumn{2}{|c|}{\begin{tabular}{l|l||}
$N$ & $N \cdot \zeta$ \\
\end{tabular}} & $N$ & $N \cdot \zeta$ & $\| N$ & $N \cdot \zeta$ & $|N|$ & $J \cdot \zeta$ & $N$ & $N \cdot \zeta$ & $N$ & $N \cdot \zeta \| N$ & $N \cdot \zeta$ & $|N|$ & $N \cdot \zeta$ \\
\hline & & 4 & $11 / 5$ & 7 & & 10 & & 13 & -32 & 16 & $\overline{31 / 10}$ & $4412 / 5$ & $\mid 22$ & 568 \\
\hline 2 & $2 / 5$ & 5 & 0 & 8 & $71 / 10$ & $\mid 11$ & & $\mid 14$ & 16 & 17 & 296|| 20 & 30 & ||23 & -148 \\
\hline 3 & 4 & 10 & & 9 & & || 12 & 12 & 15 & 340 & 18 & -112|| 21 & 428 & $\mid 24$ & 117 \\
\hline
\end{tabular}

- $K=\mathbb{Q}(\theta)$, where $\theta^{4}-4 \theta^{2}+2=0$ (discriminant 2048).

\begin{tabular}{|r|r||r|r||r|r||r|r||r|r||r|r||r|r|}
\hline$N$ & $N \cdot \zeta$ & $N$ & $N \cdot \zeta$ & $N$ & $N \cdot \zeta$ & $N$ & $N \cdot \zeta$ & $N$ & $N \cdot \zeta$ & $N$ & $N \cdot \zeta$ & $N$ & $N \cdot \zeta$ \\
\hline \hline & & 3 & 4 & 5 & -28 & 7 & 8 & 9 & -20 & 11 & 68 & 13 & -52 \\
2 & $1 / 2$ & 4 & $1 / 4$ & 6 & -10 & 8 & $9 / 4$ & 10 & 19 & 12 & $-41 / 2$ & 14 & 36 \\
\hline
\end{tabular}

\section{References}

1. A. Ash and L. Rudolph, The modular symbol and continued fractions in higher dimensions, Invent. Math. 55 (1979), 241-250.

2. J. Buchmann, D. Ford, M. Pohst, M. Olivier, and F. Diaz y Diaz, Tables of number fields of low degree, ftp://megrez.math.u-bordeaux.fr/pub/numberfields/.

3. D. S. Dummit and D. R. Hayes, Checking the $\mathfrak{p}$-adic Stark conjecture when $\mathfrak{p}$ is Archimedean, Algorithmic number theory (Talence, 1996), Springer, Berlin, 1996, pp. 91-97.

4. P. E. Gunnells, Computing Hecke eigenvalues below the cohomological dimension, J. Experimental Math. (to appear), 2000.

5. P. E. Gunnells and R. Sczech, Evaluation of Dedekind sums, Eisenstein cocycles, and special values of L-functions, preprint, 1999.

6. D. R. Hayes, Brumer elements over a real quadratic base field, Exposition. Math. 8 (1990), no. 2, 137-184.

7. $s=0$, Number theory (Banff, AB, 1988), de Gruyter, Berlin, 1990, pp. 207-226. 
8. __ Aligning Brumer-Stark elements into a Hecke character (working paper, preprint, 1998.

9. R. Sczech, Eisenstein group cocycles for $G L_{n}$ and values of L-functions, Invent. Math. 113 (1993), no. 3, 581-616.

10. L. C. Washington, Introduction to cyclotomic fields, second ed., Graduate Texts in Mathematics, no. 83, Springer-Verlag, 1997. 